\title{
Optimising broadband pulses for DEER depends on concentration and distance range of interest
}

\author{
Andreas Scherer, Sonja Tischlik, Sabrina Weickert, Valentin Wittmann, and Malte Drescher \\ Department of Chemistry and Konstanz Research School Chemical Biology, \\ University of Konstanz, Konstanz, Germany \\ Correspondence: Malte Drescher (malte.drescher@uni-konstanz.de) \\ Received: 17 February 2020 - Discussion started: 21 February 2020 \\ Revised: 15 April 2020 - Accepted: 26 April 2020 - Published: 12 May 2020
}

\begin{abstract}
EPR distance determination in the nanometre region has become an important tool for studying the structure and interaction of macromolecules. Arbitrary waveform generators (AWGs), which have recently become commercially available for EPR spectrometers, have the potential to increase the sensitivity of the most common technique, double electron-electron resonance (DEER, also called PELDOR), as they allow the generation of broadband pulses. There are several families of broadband pulses, which are different in general pulse shape and the parameters that define them. Here, we compare the most common broadband pulses. When broadband pulses lead to a larger modulation depth, they also increase the background decay of the DEER trace. Depending on the dipolar evolution time, this can significantly increase the noise level towards the end of the form factor and limit the potential increase in the modulation-to-noise ratio (MNR). We found asymmetric hyperbolic secant (HS $\{1,6\})$ pulses to perform best for short DEER traces, leading to a MNR improvement of up to $86 \%$ compared to rectangular pulses. For longer traces we found symmetric hyperbolic secant $(\mathrm{HS}\{1,1\})$ pulses to perform best; however, the increase compared to rectangular pulses goes down to $43 \%$.
\end{abstract}

\section{Introduction}

In the last few years DEER (double electron-electron resonance) has developed into an important technique for the determination of distances in the nanometre range (Jeschke, 2012; Milov et al., 1981, 1984) and in particular into a suitable tool for studying biological macromolecules (e.g. proteins (Jeschke, 2012; Robotta et al., 2014) or RNA/DNA (Grytz et al., 2017; Kuzhelev et al., 2018)). As many biomacromolecules do not contain paramagnetic centres, for many DEER experiments spin labels are introduced with the help of site-directed spin labelling (Hubbell et al., 1998). Although many different types of spin labels have been introduced in the last years, ranging from trityl (Abdullin et al., 2015; Jassoy et al., 2018), Gd(III) (Collauto et al., 2016; Dalaloyan et al., 2015; Mahawaththa et al., 2018), copper(II) (Wort et al., 2019) to photoexcitable spin labels (Di Valentin et al., 2014; Hintze et al., 2016), just to mention a few examples, nitroxide labels are still amongst the most widely used tags.
Increasing the sensitivity of DEER spectroscopy is an active field of research (Borbat et al., 2013; Breitgoff et al., 2017; Doll et al., 2015; Jeschke et al., 2004; Lovett et al., 2012; Milikisiyants et al., 2019; Polyhach et al., 2012; Tait and Stoll, 2016; Teucher and Bordignon, 2018). A very elegant approach to increasing DEER sensitivity has been made possible by the availability of arbitrary waveform generators with time resolution in the nanosecond region as they allow the generation of broadband microwave pulses (Doll et al., 2013; Doll and Jeschke, 2017; Spindler et al., 2017).

Here, we compare nitroxide-nitroxide DEER performance for different types of pulses and different sample conditions. The paper is organised as follows. In Sect. 1 we will give a brief overview of the pulse shapes that are compared in this paper. In Sect. 2, we will describe the experimental details and the compounds that have been used in this study. In Sect. 3, we will present and discuss the experimental results. We will compare rectangular, Gaussian and different types of broadband-shaped pulses on a commercial spectrometer. In 
order to give them a fair comparison, the parameters for each pulse family will be optimised. In doing so, we will provide a step-by-step guide to how an experimental optimisation for DEER can be performed. The larger inversion efficiency of broadband-shaped pump pulses that leads to a higher modulation depth will also lead to a higher background decay and therefore potentially limit the signal gain that is promised by broadband-shaped pump pulses. We set out to examine this effect for the presented pulse families in detail and show that different types of broadband-shaped pulses are ideal for different spin concentrations and distance ranges.

In magnetic resonance experiments, a pulse is generated by a time-dependent field $B_{1}$ that is applied perpendicularly to the $B_{0}$ field which defines the $z$ direction. All pulses in this paper can be described in terms of an amplitude function $A(t)$ and a frequency function $\omega(t)$.

The resulting $B_{1}$ field in the rotating frame is

$B_{1, x}(t)=A(t) \cos (\rho(t))$,

$B_{1, y}(t)=A(t) \sin (\rho(t))$,

where the phase $\rho(t)$ is defined as $\rho(t)=\int_{0}^{t} \omega\left(t^{\prime}\right) \mathrm{d} t^{\prime}$. Rectangular pulses are described by $\omega(t)=0$ and $A(t)=\left|B_{1}\right|$ during the pulse, i.e. by a $B_{1}$ field with a constant phase and intensity. The sidebands of the sinc-shaped excitation profile of rectangular pulses increase the overlap of the observer and pump pulses in DEER, resulting in so-called " $2+1$ " artefacts at the end of the DEER trace. It has recently been shown that those artefacts can be reduced by replacing the rectangular pulses with Gaussian pulses (Teucher and Bordignon, 2018). Gaussian pulses also have a frequency function of $\omega(t)=0$ but an amplitude function

$A(t)=\exp \left(-\frac{4 \ln (2) t^{2}}{\mathrm{FWHM}^{2}}\right)$.

FWHM describes the full width at half maximum of the pulse in the time domain (Teucher and Bordignon, 2018). Here and in the following equations the time axis is defined such that $t=0$ lies in the centre of the pulses. During a rectangular or Gaussian pulse the magnetisation vector is rotated around a fixed axis with an angle that is independent of the initial orientation of the magnetisation vector. Such pulses are therefore called uniform rotation pulses (Kobzar et al., 2012). As rectangular and Gaussian pulses have a fixed frequency, they are also referred to as monochromatic pulses.

One of the most significant challenges in EPR spectroscopy is the limited excitation bandwidth of rectangular and also Gaussian pulses compared to the width of many EPR spectra. In the case of nitroxide-nitroxide DEER, a significant part of the EPR spectrum contributes neither to observing nor to pumping when using rectangular pulses.

Using broadband-shaped pulses, the excitation bandwidth can be increased (Doll et al., 2013). Broadband-shaped pulses differ from rectangular and Gaussian pulses mainly in that they do not have a constant frequency, but the frequency is swept over a given range during the pulse, which allows the excitation bandwidth to be increased. In an accelerated frame, which rotates with the instantaneous excitation frequency of the pulse, the effective field rotates from the $+z$ to $-z$ directions (Baum et al., 1985; Deschamps et al., 2008; Garwood and DelaBarre, 2001; Kupče and Freeman, 1996). Under adiabatic conditions the magnetisation follows the effective field on its way from $+z$ to $-z$ (Baum et al., 1985; Doll et al., 2013). Pulses that induce this kind of spin flip behaviour are called point-to-point rotation pulses. This approach allows the generation of pulses that have a large excitation bandwidth and that are, above a certain threshold, more insensitive to the resonator profile than rectangular pulses (Baum et al., 1985). Their ability to flip spins from the $+z$ to the $-z$ axis makes such broadband-shaped pulses perfect candidates for the pump pulse in the DEER pulse sequence. Their larger excitation profile has the potential to result in a larger modulation depth and therefore a larger sensitivity (Bahrenberg et al., 2017; Doll et al., 2015; Spindler et al., 2013; Tait and Stoll, 2016).

Intuitively, a high adiabaticity means that the effective magnetic field moves more slowly from $+z$ to $-z$, making it easier for the spins to follow, thus resulting in a higher inversion efficiency.

The adiabaticity $Q$ is formally defined as (Kupče and Freeman, 1996)

$Q=\frac{2 \pi v_{\mathrm{eff}}}{|\mathrm{d} \theta / \mathrm{d} t|}$.

Here, $v_{\text {eff }}$ is the strength of the effective magnetic field and $\theta$ is its polar angle in the accelerated frame. The pulses have a good inversion efficiency, if $Q \gg 1$ (Deschamps et al., 2008). In general, the adiabaticity changes during the duration of the pulse and is different for spins with different frequency offsets. Adiabatic pulses are typically quantified by their minimum adiabaticity $Q_{\min }$.

Chirp pulses have a constant amplitude function and a linear frequency function $\omega(t)=f_{\text {start }}+p t$, where $p=\Delta f / t_{\mathrm{p}}$ is a sweep constant, $t_{\mathrm{p}}$ is the pulse length and $\Delta f=f_{\text {end }}-f_{\text {start }}$. $f_{\text {start }}$ and $f_{\text {end }}$ are the start and end frequencies of the frequency sweep. The minimum adiabaticity $Q_{\min }$ is reached when a spin is on resonance with the pulse frequency (Doll et al., 2013):

$Q_{\min }=\frac{2 \pi v_{1}^{2} t_{\mathrm{p}}}{\Delta f}$,

where $v_{1}$ is the nutation frequency which increases linearly with $\left|B_{1}\right| . Q_{\text {min }}$ increases with the pulse length but decreases with the sweep width. The frequency width for a pump pulse should be chosen such that a large part of the spectrum is excited without having significant spectral overlap with the pulses at the observer frequency. The steep flanks at the beginning and the end of the rectangular amplitude profile lead 
to distortions in the excitation profiles of chirp pulses, because the initial effective magnetic field is not aligned with the $z$ axis. Smoothing both ends of the pulses with a quarter sine wave can reduce theses distortions (Böhlen and Bodenhausen, 1993). The smoothing can be adapted by changing the rising time $t_{\text {rise }}$. Following the logic so far, the pulse length should be chosen as long as possible to enable a very high adiabaticity. However, a broadband-shaped pulse flips spins with different offsets at different times. When used as a pump pulse in DEER, this results in a shift of the dipolar oscillations and an artificial broadening of smaller distances in the distance distribution. Therefore, the pulse length should be chosen such that (Breitgoff et al., 2019)

$t_{\mathrm{p}}<\frac{T_{\mathrm{dd}}}{4}$,

with the dipolar evolution time $T_{\mathrm{dd}}$ of the shortest expected distance.

In addition to chirp pulses, there are more elaborate pulses employing more elaborate frequency and amplitude functions. The most common ones are WURST (wideband, uniform, smooth truncation) and HS (hyperbolic secant) pulses. The trends discussed so far are valid for them as well. However, they feature additional parameters that can be used to tune the steepness of the corresponding excitation profiles.

WURST pulses have a linear frequency sweep as well but a different amplitude function than chirp pulses (Kupče and Freeman, 1995b; Spindler et al., 2017):

$A(t)=A_{\max }\left(1-\left|\sin \frac{\pi t}{t_{\mathrm{p}}}\right|^{n}\right)$.

The effect of the parameter $n$ determining the steepness of the amplitude function will be discussed below.

HS pulses have non-linear frequency sweeps and are described by the following amplitude and frequency functions:

$$
\begin{aligned}
& A(t)=\operatorname{sech}\left(\beta 2^{h-1}\left(\frac{t}{t_{\mathrm{p}}}\right)^{h}\right), \\
& \omega(t)=\frac{\Delta f}{2} \tanh \left(\frac{\beta}{2}\right)^{-1} \tanh \left(\frac{\beta t}{t_{\mathrm{p}}}\right),
\end{aligned}
$$

with order parameter $h$ and truncation parameter $\beta$. The effects of $\beta$ will be discussed below. A common choice for $h$ is to set $h=1$. These pulses have an offset-independent adiabaticity and a rather rectangular excitation profile (Baum et al., 1985; Tannús and Garwood, 1996). Increasing the order $h$ of an HS pulse will lead to a higher adiabaticity at the maximum of the excitation profile but less steep flanks (Breitgoff et al., 2019). A compromise can be found by using an asymmetric HS pulse where the flank close to the observer is made steep by an order of 1 and where the other flank has a higher order for a higher adiabaticity (Doll et al., 2016). Symmetric pulses with an order parameter of $h=1$ will be referred to as $\mathrm{HS}\{1,1\}$; asymmetric pulses where the first part of the pulse has an order parameter of $h=1$ and the second half has $h=6$, as suggested by Doll et al. (2016), are referred to as HS $\{1,6\}$ (Doll et al., 2016).

The measured DEER trace $V(t)$ is the product of the form factor $F(t)$ that contains the required intramolecular distance information and a background function $B(t)$ (Jeschke, 2012):

$V(t)=F(t) \cdot B(t)$.

The background decay is caused by the intermolecular interactions of the observer spin with pump spins of surrounding molecules. Assuming that the spins are homogenously distributed, the background decay can be described by an exponential decay (Jeschke, 2016):

$B(t)=\exp \left(-(k|t|)^{d / 3}\right)$,

where $d$ is a dimensionality constant and the decay constant $k$ is described by the following equation (Hu and Hartmann, 1974; Pannier et al., 2000):

$k=\frac{2 N_{\mathrm{a}} \pi \mu_{0}}{9 \sqrt{3} h} g^{2} \mu_{e}^{2} f c$.

Here, $c$ is the spin concentration, $f$ the inversion efficiency of the pump pulse, $\mu_{e}$ the Bohr magneton, $\mu_{0}$ the magnetic field constant, $N_{\mathrm{a}}$ the Avogadro number and $g$ the isotropic $g$ factor of the nitroxide.

\section{Materials and methods}

\subsection{Sample preparation}

Wheat germ agglutinin (WGA) was purchased from SigmaAldrich (article-no.: L9640) as lyophilised powder and used without further purification. The doubly spin-labelled tetravalent ligand (1) was synthesised in the lab of Valentin Wittmann. Details of synthesis and characterisation can be found in Rohse et al. (2020)). For the WGA-ligand samples investigated in this study solutions of WGA and the tetravalent ligand were prepared separately in deionised water. The protein concentration of the WGA solution was determined spectrophotometrically.

WGA-ligand samples were prepared by mixing WGA and ligand solutions resulting in a $2: 1$ molar excess of WGA compared to the ligand referring to the final sample volume. The 2-fold excess on protein was chosen to prevent a free, unbound ligand in solution. The sample solution was lyophilised and the resulting powder was dissolved in D2O (Magnisolv, Cas-no.: 7789200, article: S571556621) and $20 \%(v / v)$ deuterated glycerin (Sigma-Aldrich, lotno. MBBB5255, article: $447498-1 G$ ) as cryoprotectant. Unless stated otherwise we used a sample concentration of $160 \mu \mathrm{M}$ WGA and $80 \mu \mathrm{M}$ ligand; $60 \mu \mathrm{L}$ of solution was filled into $3 \mathrm{~mm}$ outer diameter quartz sample tubes (ER 221 TUB/2, part no. E221003), shock-frozen in liquid nitrogen before measurement and placed in the probe head precooled to $50 \mathrm{~K}$. Samples were stored at $-80^{\circ} \mathrm{C}$ with unfreezing avoided. 


\subsection{EPR experiments}

All experiments have been performed on a Bruker Elexsys E580 spectrometer at $Q$ band $(34 \mathrm{GHz})$. The spectrometer is equipped with a SpinJet-AWG unit (Bruker) and a $150 \mathrm{~W}$ pulsed travelling-wave tube (TWT). All samples were measured in $3 \mathrm{~mm}$ outer diameter sample tubes in an overcoupled ER5106QT-2 resonator (Bruker). The quality factor $Q$ of the overcoupled resonator is approximately 200.

The samples were cooled to $50 \mathrm{~K}$ with a Flexline helium recirculation system (CE-FLEX-4K-0110, Bruker Biospin, ColdEdge Technologies) comprising a cold head (expander, SRDK-408D2) and an F-70H compressor (both SHI cryogenics, Tokyo, Japan), controlled by an Oxford Instruments Mercury ITC.

DEER measurements were recorded with the standard four-pulse DEER sequence (Pannier et al., 2000), an eightstep phase cycle (Tait and Stoll, 2016) and nuclear modulation averaging (Jeschke, 2012). The dipolar evolution time was set to $8 \mu$ s and the time step to $8 \mathrm{~ns}$.

We analysed the DEER traces with DeerAnalysis2019 (Jeschke et al., 2006). We performed a background correction resulting in a background function with a dimension of $d=3.5$. The form factor was analysed with Tikhonov regularisation and a regularisation parameter chosen by the generalised cross-validation criterion (Edwards and Stoll, 2018).

A crucial parameter for pulsed dipolar spectroscopy is the modulation-to-noise parameter $\mathrm{MNR}=\frac{\lambda}{n}$, with the modulation depth $\lambda$ and the noise level $n$. We calculated the noise similarly to published procedures by the standard error from a fit with a smoothing spline (Bahrenberg et al., 2017; Breitgoff et al., 2019; Mentink-Vigier et al., 2013). We excluded the first 10 data points from the form factor because the spline typically showed some deviations at the start of the trace. Unless stated otherwise, the upper limit for the noise calculation was $7 \mu \mathrm{s}$.

We used the $\eta_{2 P}$ parameter which has been suggested by Doll et al. (2015) and already been used by other authors (Doll et al., 2015; Spindler et al., 2013; Tait and Stoll, 2016). The $\eta_{2 P}$ value is defined as the difference between two distinct time points in the DEER trace and therefore does not require the measurement of full DEER traces. We recorded short DEER traces with eight data points only and calculated $\eta_{2 P}$ as the difference of the phase-corrected DEER trace at the zero time $V(0)$ minus the first minimum of the DEER trace $V\left(t_{\min }\right)$.

For a more detailed description of materials and methods, see Sect. S1 in the Supplement.

\section{Results and discussion}

In order to study the performance of DEER using different pulses, we used the doubly nitroxide-labelled tetravalent ligand bound to wheat germ agglutinin dimer (WGA) as a model system (Fig. 1). The ligand binds with a very high affinity to WGA and features a narrow distance distribution $(\mathrm{FWHM}=0.2 \mathrm{~nm})$ at $5.1 \mathrm{~nm}$ (Rohse et al., 2020). We performed DEER experiments with different combinations of pulses. In the following, we will refer to a combination of rectangular observer and pump pulses as RR, to a combination of Gaussian observer and pump pulses as GG, to a combination of rectangular observer and broadband-shaped pump pulses as RS and to a combination of Gaussian observer and broadband-shaped pump pulses as GS.

As stated by Eq. (10), the measured raw data not only consist of the desired form factor, but also include a background contribution emerging from intermolecular interactions. A common way to deal with this is to fit the background according to Eq. (11) and divide the raw data by the fit to obtain the form factor that can then be transformed into a distance distribution (Jeschke, 2012; Jeschke et al., 2006). When measuring DEER traces, a precise distance determination is desired. Since for an experimental parameter optimisation the true underlying distance distribution is unknown, a metric is needed that is based on the recorded data. The MNR of the form factor is suitable for this case as it increases with an increasing modulation depth and an increasing echo intensity. As the noise of the form factor increases towards its end due to the division by the background, the MNR goes down with a stronger background decay. It can therefore capture the fact that a larger background decay leads to less reliable distance distributions, as has recently been investigated by Fábregas Ibáñez and Jeschke (2020) in a detailed study. In their paper they also suggest a different method for background correction that treats the background by directly including it in the kernel that is needed to calculate the distance distribution from the DEER trace. As this methods renders the calculation of a form factor redundant, a MNR cannot be directly obtained by it. Even though this new method has shown itself to give more reliable distance distributions in the case of large background decays, its performance still drops with an increasing background. Therefore, we still consider the MNR that is obtained by the background correction by division to be the best measure to optimise settings for DEER measurements experimentally.

The evaluation of the noise of the entire DEER trace is not always feasible. It depends on the maximum distance $r_{\max }$ that is to be detected up to which part the form factor is of interest. Here, we truncated the form factor for the calculation of the MNR at 3 times the oscillation period of the maximum distance that is of interest (Edwards and Stoll, 2018):

$\tau_{\text {truncation }}=3 \frac{\left(\frac{r_{\max }}{n m}\right)^{3}}{52 \mathrm{MHz}}$.

This corresponds to roughly three dipolar oscillations in the form factor. In this case of a distance at $5.1 \mathrm{~nm}$ this is equivalent to a truncation time $\tau_{\text {truncation }} \approx 7 \mu \mathrm{s}$. A simulation with a model distance reveals that in order to obtain the correct width of the distance distribution, a good MNR up to this time point can be necessary and a good MNR of only the 

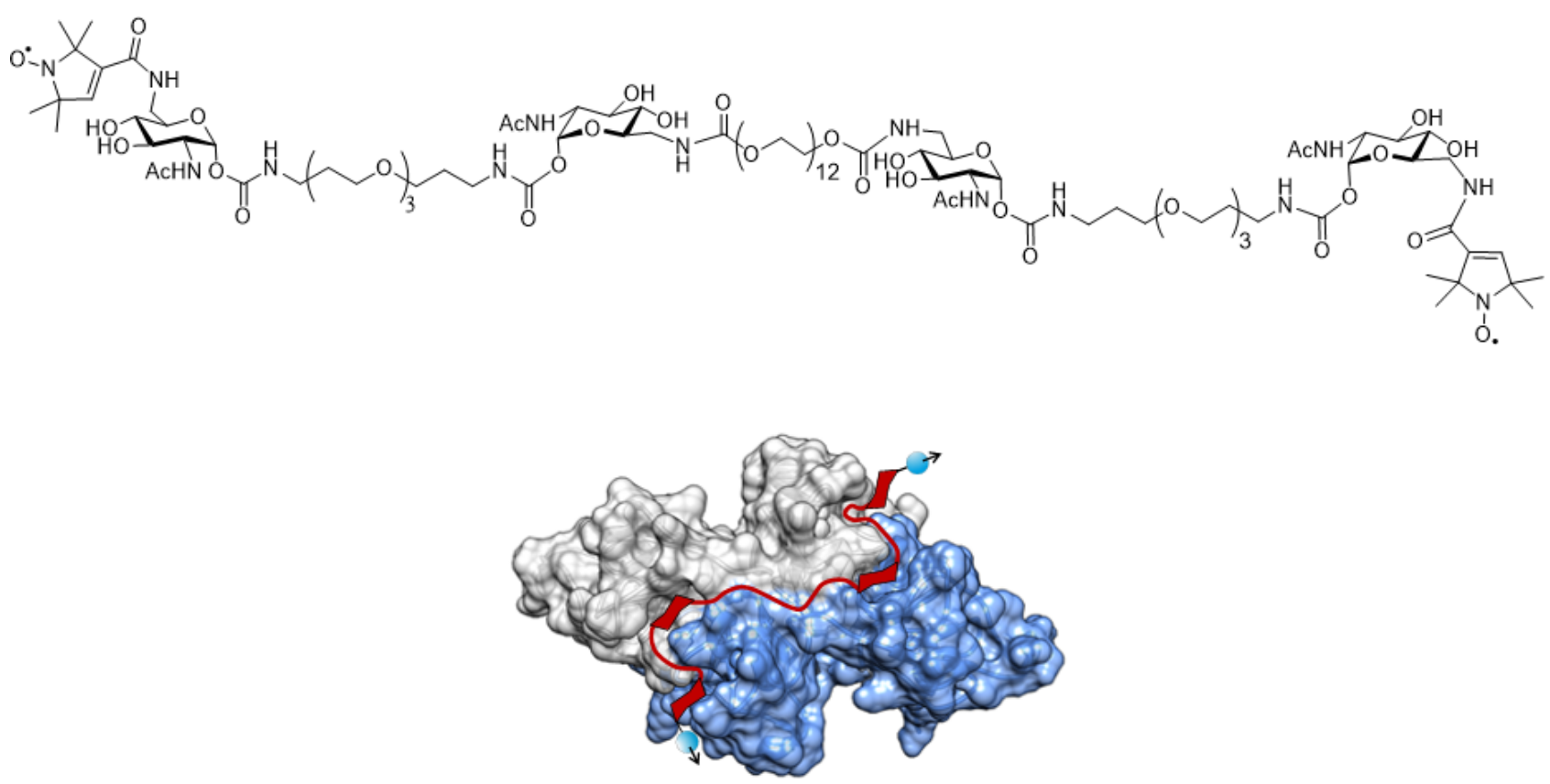

Figure 1. The structure of the tetravalent ligand with its two spin-(2,2,5,5-tetramethyl-3-pyrrolin-1-yloxycarbonyl) labels and the ligand bound to WGA. The visualisation of the dimeric WGA with the subunits coloured in grey and blue is based on the crystal structure (PDB entry 2X52; Schwefel et al., 2010). In red, a schematic representation of the ligand is overlaid to the crystal structure. The ligand is suggested to bind with its four GlcNAc moieties to the primary binding sites of WGA. Blue balls and arrows indicate nitroxide spin labels.

first part of the form factor is not as reliable when the credibility of the obtained distance distribution is to be estimated. The details of this study can be found in Sect. S2.

\subsection{Performance comparison for rectangular and Gaussian pulses}

The pump pulse frequency was set to $34.00 \mathrm{GHz}$, which is the maximum of the resonator profile (Fig. 2a). The magnetic field was set such that we pumped on the maximum of the nitroxide spectrum (Fig. 2b). To optimise the settings for RR and GG we tested observer pulses with a frequency offset of 90 and $70 \mathrm{MHz}$ between the pump and observer pulses, respectively. To check for different excitation profile widths of the observer pulse, we tested settings with observer pulse amplitudes of $100 \%$ and $60 \%$. The pulse length was always adjusted to get $\pi / 2$ and $\pi$ pulses. The observer pulse lengths for both tested frequency offsets were identical in all experiments owing to the similar values of the resonator profile at both observer frequencies (33.91 and $33.93 \mathrm{GHz}$, Fig. 2a). For rectangular observer pulses the pulse lengths were 28 and $32 \mathrm{~ns}$; for Gaussian observer pulses, they were 56 and $74 \mathrm{~ns}$. For the pump pulse we kept the amplitude fixed at $100 \%$, which resulted in pulse lengths of $16 \mathrm{~ns}$ for rectangular and $34 \mathrm{~ns}$ for Gaussian pulses. As we used Gaussian pulses that were generated by Xepr, the FWHM of the Gaussian pulses was automatically defined by the software as $\mathrm{FWHM}=t_{\mathrm{p}} / 2 \sqrt{2} \ln (2)$, and we did not optimise this param-
Table 1. The rectangular and Gaussian pulses with the best performance.

\begin{tabular}{lrrrr}
\hline $\begin{array}{l}\text { Pulse } \\
\text { Type }\end{array}$ & $\begin{array}{r}\text { Offset } \\
(\mathrm{MHz})\end{array}$ & $\begin{array}{r}\text { Obs. amp. } \\
(\%)\end{array}$ & MNR & $\begin{array}{r}\text { Mod. depth } \\
\lambda\end{array}$ \\
\hline RR & 70 & 60 & 35 & 0.31 \\
GG & 70 & 100 & 41 & 0.31 \\
\hline
\end{tabular}

eter. An overview of all observer pulse settings can be found in Tables S1 and S2 in the Supplement.

For optimum observation of the spin echo modulation in DEER traces, it has been suggested to record the echo in transient mode and then perform a digital integration over a product of the recorded echo with a Gaussian filter (Pribitzer et al., 2017). This procedure is not ideal for commercial spectrometers as the transient recording of the echo drastically increases the spectrometer overhead time. Therefore, we performed a direct integration of the spin echo. We optimised the integration window for each parameter set for a maximum MNR by recording a series of Hahn echoes. Compared with commonly used integration lengths equal to the $\pi$-pulse length for rectangular pulses (Jeschke, 2007), we find settings where a $14 \%$ increase in the SNR can be achieved by choosing a larger integration window. For Gaussian pulses, we find that it is typically preferable to choose integration windows that are shorter than the $\pi$-pulse length. More details can be found in Sect. S2. 

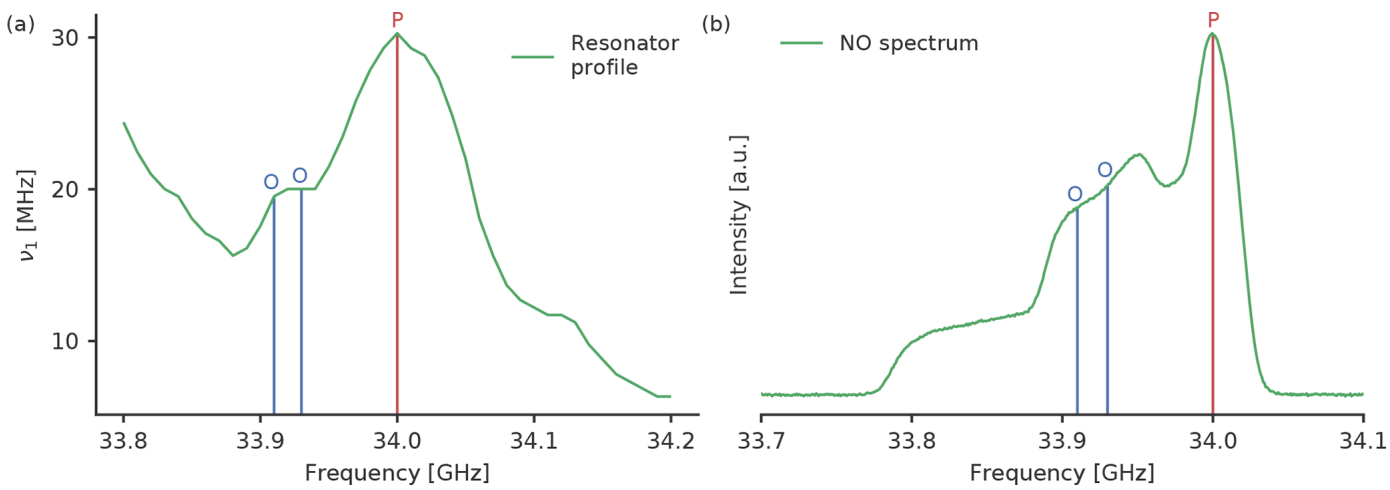

Figure 2. (a) Resonator profile with both tested observer frequencies (blue) and the pump (red) frequencies for the rectangular pulses and (b) the nitroxide spectrum with the positions of the two tested observer frequencies and the pump frequency.

The best MNR for the setting RR was found to be 35 (Table 1). It was achieved for an offset of $70 \mathrm{MHz}$ and $60 \%$ intensity. The best MNR for GG was 41 at an offset of $70 \mathrm{MHz}$ as well and a pulse amplitude of $100 \%$ (Table 1). This corresponds to a $17 \%$ increase in the MNR of Gaussian pulses compared to rectangular pulses. This is in contrast to the findings of Teucher and Bordignon (2018), who found that Gaussian observer pulses have a slightly lower MNR that rectangular pulses. The exact reason for the deviating results is not entirely clear to us: we assume that this is due to their different setup with a homebuilt resonator that has slightly different properties than our commercial one.

As expected, the missing sidebands of the Gaussian pulses allow the usage of higher pulse amplitudes. This hints that for the chosen parameters the pulse overlap is indeed a limiting factor for rectangular pulses. The modulation depth for RR and GG is approximately $30 \%$ in both cases, but Gaussian pulses seem to have the advantage of a higher echo intensity, probably due to a lower pulse overlap. For RR and GG, the small offset of $70 \mathrm{MHz}$ performed better than a larger offset of $90 \mathrm{MHz}$, most likely due to the different echo intensities at the corresponding positions in the EPR spectrum (Fig. 2b). The results for all RR and GG settings can be found in Tables S3 and S4.

\subsection{Broadband-shaped pulses}

We set out to investigate several broadband-shaped pulses, i.e. chirp, WURST, $\operatorname{HS}\{1,1\}$ and $\operatorname{HS}\{1,6\}$ pulses for the settings RS and GS. Unless specified otherwise, we used pump pulse lengths of $100 \mathrm{~ns}$. According to Eq. (6), the pulse length of $100 \mathrm{~ns}$ corresponds to a minimum accessible distance of $r_{\min }=2.75 \mathrm{~nm}$. For the determination of shorter distances we also tested chirp pulses with a length of $36 \mathrm{~ns}$ (referred to as short chirp pulses below), which corresponds to a distance of $r_{\min }=1.96 \mathrm{~nm}$. Such a distance limit should be suitable for most practical applications. Despite the fact that longer broadband-shaped pump pulses should give higher inversion efficiencies, we found that they do not result in a better per- formance for DEER. As the minimum accessible distance also increases when longer pump pulses are used, we did not test pump pulses longer than $100 \mathrm{~ns}$. This is discussed in more detail in Sect. S13.

Spins are not flipped within the whole pulse duration, but only a smaller fraction of it (Spindler et al., 2013). Simulations with an $\operatorname{HS}\{1,1\}$ pulse with a length of $t_{\mathrm{p}}=100 \mathrm{~ns}$, a frequency sweep width of $\Delta f=110 \mathrm{MHz}$ and $\beta=8 / t_{\mathrm{p}}$ show distances up to $r_{\min }=2.32 \mathrm{~nm}$ could be detectable ( $\mathrm{see}$ Sect. S7). It is, however, hard to generalise this effect as the spin trajectories for different broadband pulses are not necessarily the same.

Figure 3 shows the calculated excitation profiles of some of the tested pulses. The calculated excitation profiles are normalised to a $v_{1}$ field strength of $30 \mathrm{MHz}$, which we achieved with our setup at the maximum of the resonator profile. Under such conditions, the long chirp pulses have an adiabaticity of around 5, i.e. a chirp pulse with a length of $100 \mathrm{~ns}$, a sweep width of $120 \mathrm{MHz}$ and a $v_{1}$ strength of $30 \mathrm{MHz}$ with a calculated adiabaticity of 4.7. A short chirp pulse with a length of $36 \mathrm{~ns}$ (and otherwise unchanged parameters) has an adiabaticity of 1.7 due to the higherfrequency sweep rate. Although this value is rather low, the calculations show that short chirp pulses achieve a nearly complete inversion efficiency around the maximum of the excitation profile (Fig. 3a). On the other hand, the excitation profile is rather broad, with many sidebands. The finite length of the pulses creates an additional distortion. By smoothing the edge with a quarter sine, this disturbance can be reduced (Fig. 3a). A higher rising time will lead to a more properly defined excitation profile with fewer sidebands, but the overall width of the excitation profile is reduced (see Fig. 3a).

WURST pulses (Fig. 3b) are characterised by an additional parameter $n$. A high value of $n$ results in a more rectangular shape of the pulse and leads to distortions in the excitation profile around the maximum (Kupče and Freeman, 1995a, b; O'Dell, 2013). Small values of $n$ lead to excitation profiles with very steep and well-defined side flanks. 

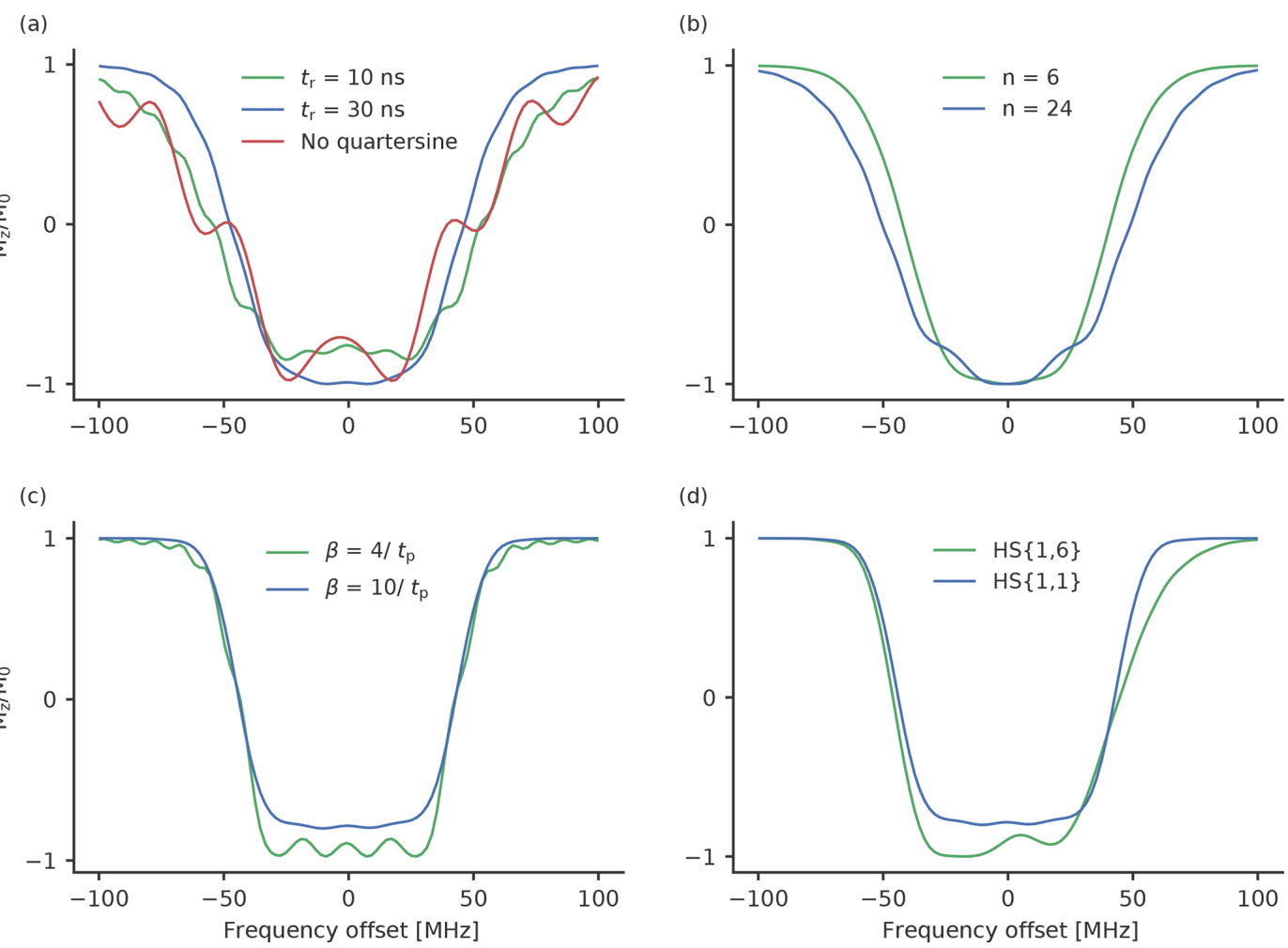

Figure 3. Calculated inversion profiles of broadband pulses normalised to $v_{1}=30 \mathrm{MHz}$, which corresponds to the maximum of the measured resonator profile. (a) Chirp pulses with a frequency width of $120 \mathrm{MHz}$, a length of $100 \mathrm{~ns}$ and a rising time of $10 \mathrm{~ns}$ (green), a rising time of $30 \mathrm{~ns}$ (blue), a length of $36 \mathrm{~ns}$ and no quarter sine smoothing (red). (b) WURST pulses with a frequency width of $120 \mathrm{MHz}$, a pulse length of $100 \mathrm{~ns}$ and a value for $n$ of 6 (green) and 24 (blue). (c) HS\{1,1\} pulses with a frequency width of $90 \mathrm{MHz}$ and truncation parameters of 4 (green) and 10 (blue). (d) An HS $\{1,6\}$ (green) and HS $\{1,1\}$ (blue) pulse with a width of $90 \mathrm{MHz}$ and a pulse length of $100 \mathrm{~ns}$. The truncation parameter was 10 in both cases.

However, for small $n$ very long pulse durations are needed to achieve a high inversion efficiency. As long pulses are not feasible, because they limit the minimum distances that can be resolved, we chose to stick to $100 \mathrm{~ns}$ pulses and test the values for $n$ of 6,12 and 24, for which a reasonable excitation profile can be expected (Fig. 3b).

In Fig. $3 \mathrm{c}$ we show the comparison of the excitation profile of $\mathrm{HS}\{1,1\}$ pulses for truncation parameters of $\beta=4 / t_{\mathrm{p}}$ and $\beta=10 / t_{\mathrm{p}}$. For $\beta=10 / t_{\mathrm{p}}$ the inversion efficiency is smaller than for $\beta=4 / t_{\mathrm{p}}$; however, the excitation profile is well defined and does not show the sideband oscillations that can be seen for the latter.

Owing to their higher adiabaticity, $\operatorname{HS}\{1,6\}$ pulses feature higher inversion efficiency than $\mathrm{HS}\{1,1\}$ pulses with otherwise equal parameters (Fig. 3d) while maintaining the steep frequency flank towards the observer profile at the lowerfrequency end.

For $\operatorname{HS}\{1,1\}$ and $\operatorname{HS}\{1,6\}$ pulses a frequency sweep width of 50 to $110 \mathrm{MHz}$ was tested. WURST and chirp pulses tend to have narrower excitation profiles for a given frequency sweep width at the tested parameters (see Fig. 4). We therefore chose to use higher-frequency sweep widths for

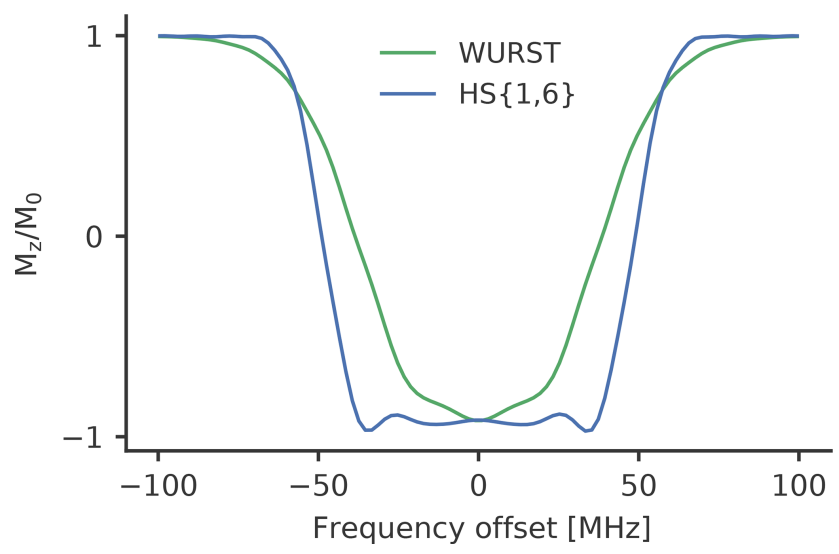

Figure 4. Calculated inversion profiles of a WURST $(n=12$, green) and a $\operatorname{HS}\{1,6\}$ (truncation parameter of $6 / t_{\mathrm{p}}$, blue) pulse with a pulse length of $100 \mathrm{~ns}$ and a sweep width of $100 \mathrm{MHz}$ normalised to $v_{1}=30 \mathrm{MHz}$, which corresponds to the maximum of the measured resonator profile. 
WURST than for HS pulses to achieve a similar excitation bandwidth.

As the bandwidth of the resonator and the width of the spectrum are limited, there is an optimum offset between the two pulses that minimises the overlap but is not too large for the resonator bandwidth. We tested offsets from a range of 70 to $130 \mathrm{MHz}$. The offset is defined as the difference between the observer frequency and the centre of the frequency sweep of the broadband-shaped pulses. For the optimisation measurements, the frequency of the observer channel was fixed and the frequency of the pump pulse was changed stepwise. We shifted the magnetic field with the pump pulse so that we always pumped on the maximum of the spectrum (see Sect. S1). During the increase in the offset, the position of the observer pulses in the spectrum will change as the spectrum is shifted, with the pump pulse resulting in a decrease in the echo for higher offsets. Table 2 shows an overview of all tested pump pulse parameter sets.

We used the same parameters for the observer pulses as before, meaning that we tried rectangular and Gaussian observer pulses at microwave frequencies of 33.91 and $33.93 \mathrm{GHz}$ at $100 \%$ and $60 \%$ amplitude, respectively (see Tables S1 and S2), and combined them with all the broadband-shaped pulses from Table 2. This results in 504 different settings (Table 2) for the pump pulse and 8 different settings for the observer pulses, which gives a total of 4032 different DEER settings. As the measurement of full DEER traces and subsequent determination of the MNR would be very time-consuming, we used the $\eta_{2} P$ parameter as an estimation for the MNR. This was suggested by Doll et al. (2015) and already used by other authors (Spindler et al., 2013; Tait and Stoll, 2016). As it requires only two points of the DEER trace, the measurement time can be drastically reduced. However, it has the disadvantage that artefacts, e.g. echo-crossing artefacts or nuclear modulation, might remain undetected. Therefore, we decided to additionally perform phase cycling and nuclear modulation averaging. For different observer pulse settings, the $\eta_{2 P}$ parameters are not necessarily comparable, because $\eta_{2 P}$ assumes a constant absolute noise level. However, this noise level could change with different integration windows. Hence, we identified the best chirp, WURST, HS $\{1,1\}$ and HS $\{1,6\}$ pulse for each observer pulse setting and recorded full DEER traces of them, giving a total number of 16 traces of types RS and GS each.

Exemplary heat maps showing the $\eta_{2 P}$ for Gaussian observer pulses and $100 \mathrm{~ns}$ pump pulses can be found in Fig. 5 . We could identify several trends that were true for all observer pulse settings. HS $\{1,1\}$ and $\operatorname{HS}\{1,6\}$ pulses have higher maximum $\eta_{2 P}$ values than chirp and WURST pulses. HS $\{1,1\}$ and $\operatorname{HS}\{1,6\}$ have their highest $\eta_{2 P}$ values for smaller offsets than chirp and WURST pulses. This fits to the steeper flanks in their excitation profiles and a resulting smaller overlap with the observer frequency. Nonetheless, the overall range of reasonable offsets for all pulses is rather small and within a range of 80 and $100 \mathrm{MHz}$, meaning that for nitroxide-nitroxide DEER and our setup the width of the spectrum and the resonator profile has a more crucial influence in choosing the right offset than the excitation profiles of the different pump pulses. HS $\{1,1\}$ and $\operatorname{HS}\{1,6\}$ pulses have smaller ideal frequency widths of 90 and $110 \mathrm{MHz}$, whereas for chirp and WURST pulses the frequency widths seem to be ideal at 120 and $160 \mathrm{MHz}$. This fits to the already mentioned observation that WURST pulses have smaller excitation profiles for a given sweep width with the used parameters than $\operatorname{HS}\{1,1\}$ and HS $\{1,6\}$ pulses (see Fig. 4). Interestingly, despite their lower adiabaticity, the short chirp pulses with a length of $36 \mathrm{~ns}$ had a larger $\eta_{2 P}$ value than the chirps with a length of $100 \mathrm{~ns}$ for all observer pulse settings. Quarter sine smoothing does not necessarily lead to a better performance of the short chirp pulses. For the WURST pulses, a value of $n=6$ gives the best performance with all observer pulses. For different observer pulses, we find that the best performance of $\operatorname{HS}\{1,1\}$ and $\operatorname{HS}\{1,6\}$ pulses can be achieved with $\beta$ parameters ranging from $6 / t_{\mathrm{p}}$ to $10 / t_{\mathrm{p}}$.

For all observer pulse settings, we identified the best parameter set for each pulse family, resulting in a maximised $\eta_{2 P}$. We then recorded a full DEER trace for each family and compared them by their MNRs. All results for the full DEER traces can be found in Tables S5 and S6. Table 2 shows the parameters and observer pulses that resulted in the best performing chirp, WURST, HS $\{1,1\}$ and $\operatorname{HS}\{1,6\}$ pulses for the full DEER traces. We found that also for broadbandshaped pump pulses Gaussian observer pulses outperform rectangular ones. Again, this hints that Gaussian observer pulses can successfully reduce the frequency overlap with the pump pulse due to their missing sidebands. In all scenarios we found that an observer pulse that is positioned with a $70 \mathrm{MHz}$ offset to the maximum of the resonator profile performs better than an observer pulse position with a $90 \mathrm{MHz}$ offset to the maximum of the resonator profile. The offset to the broadband-shaped pump pulse, however, does not change on average, which means that in the former case the observer and pump pulses have a more symmetric positioning around the maximum.

Broadband-shaped pump pulses lead to a larger modulation depth than rectangular and Gaussian pulses. Whereas for non-broadband pulses the modulation depth is limited to around $30 \%$ with our setup, we achieved an increase of up to $63 \%$ with WURST pulses. HS $\{1,6\}$ pulses also lead to high modulation depths of $61 \%$. For chirp and HS $\{1,1\}$ pulses smaller modulation depths of approx. $50 \%$ were observed. However, the highest modulation depth will not necessarily lead to the highest MNR, as can be seen in Table 3. This is due to a larger background decay of pulses with a higher inversion efficiency and will be analysed in the next section. Due to a higher bandwidth overlap, broadband-shaped pulses will also reduce the echo intensity more strongly than rectangular or Gaussian pulses. HS $\{1,1\}$ pulses seem to be a good compromise between a high modulation depth, a high echo intensity and a background decay that is not too steep. 
Table 2. The parameters for the broadband-shaped pump pulses.

\begin{tabular}{lrlll}
\hline Pulse type & Length (ns) & Frequency width $(\mathrm{MHz})$ & Offset $(\mathrm{MHz})$ & additional parameter \\
\hline chirp & 100 & $80,120,160200$ & $70-130$ & $t_{\mathrm{r}}=t_{\mathrm{p}} / 4,10,30 \mathrm{~ns}$ \\
short chirp & 36 & $80,120,160,200$ & $70-130$ & $t_{\mathrm{r}}=t_{\mathrm{p}} / 4,10,30 \mathrm{~ns}$ and without quarter sine smoothing \\
WURST & 100 & $80,120,160,200$ & $70-130$ & $n=6,12,24$ \\
$\mathrm{HS}\{1,1\}$ & 100 & $50,70,90,110$ & $70-130$ & $\beta=4 / t_{\mathrm{p}}, 6 / t_{\mathrm{p}}, 8 / t_{\mathrm{p}}, 10 / t_{\mathrm{p}}$ \\
$\mathrm{HS}\{1,6\}$ & 100 & $50,70,90,110$ & $70-130$ & $\beta=4 / t_{\mathrm{p}}, 6 / t_{\mathrm{p}}, 8 / t_{\mathrm{p}}, 10 / t_{\mathrm{p}}$ \\
\hline
\end{tabular}
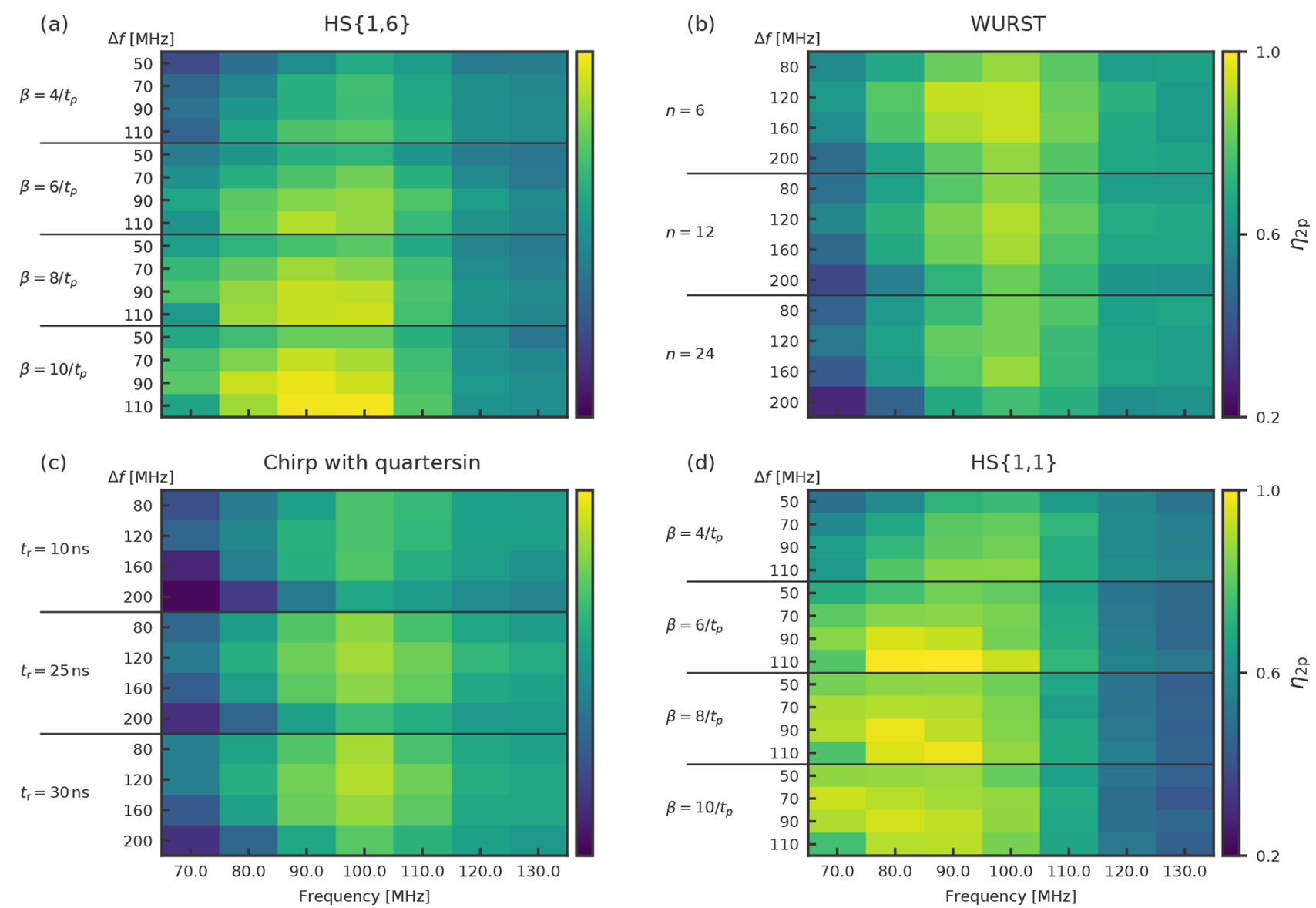

Figure 5. Heat maps with the $\eta_{2 P}$ values for 4p-DEER measurements with an observer pulse length of $56 \mathrm{~ns}$, Gaussian pulses and an integration window of $56 \mathrm{~ns}$. The observer frequency is at $33.93 \mathrm{GHz}$. The pump pulse length is $100 \mathrm{~ns}$. Each heat map shows a different pump pulse type: (a) HS $\{1,6\}$, (b) WURST, (c) chirp with quarter sine, and (d) HS $\{1,1\}$.

They resulted in the highest MNR of 50 with a pulse length of $100 \mathrm{~ns}$, an offset of $90 \mathrm{MHz}$, a frequency bandwidth of $110 \mathrm{MHz}$ and $\beta=8 / t_{\mathrm{p}}$ with the observer pulses being Gaussian pulses with an amplitude of $100 \%$ and a frequency of $33.93 \mathrm{GHz}$. Interestingly, this performance is achieved although the broadband pulse does not achieve a complete inversion (Fig. S8d in the Supplement). The modulation depth in that case increased to $52 \%$ (Fig. S11). This corresponds to an MNR increase of $43 \%$ compared to RR and $22 \%$ compared to GG. To estimate the lower limit of distances that can be determined with such a $100 \mathrm{~ns}$ pulse, we performed a simulation to see when the spins are actually flipped during the experiment (see Sect. S7). A visual inspection reveals that most spins are flipped between 20 and $80 \mathrm{~ns}$ within the pulse duration, making it an effective length of $60 \mathrm{~ns}$ where the spin flips occur, which would correspond to a minimum detectable distance limit of $2.3 \mathrm{~nm}$ instead of $2.8 \mathrm{~nm}$ for a 100 ns spin-flip period.

Depending on the resonator and the microwave amplifier, different $B_{1}$ field strengths are available on different spectrometers. However, as the inversion efficiency of broadbandshaped pulses is less dependent on the $B_{1}$ field strength, as is 
Table 3. The parameters of the observer and pump pulses that gave the best MNR for each pump pulse type. All observer pulses are Gaussian pulses with a pulse length of $74 \mathrm{~ns}$ for a $60 \%$ intensity and $56 \mathrm{~ns}$ for a $100 \%$ intensity. The observer frequency was $33.93 \mathrm{GHz}$ in all cases. The MNR was evaluated up to $7 \mu$ s.

\begin{tabular}{lrrrrrr}
\hline Pump pulse & $\begin{array}{r}\text { Obs. } \\
\text { Amp. }(\%)\end{array}$ & $\begin{array}{r}t_{\pi} \\
(\mathrm{ns})\end{array}$ & $\begin{array}{r}\Delta f \\
(\mathrm{MHz})\end{array}$ & $\begin{array}{r}\text { Offset } \\
(\mathrm{MHz})\end{array}$ & MNR & Mod depth $\lambda$ \\
\hline HS $\{1,6\}\left(\beta=10 / t_{\mathrm{p}}\right)$ & 60 & 100 & 110 & 90 & 45 & 0.61 \\
WURST $(n=6)$ & 60 & 100 & 160 & 90 & 40 & 0.63 \\
Chirp (no smoothing) & 100 & 36 & 120 & 80 & 45 & 0.49 \\
$\operatorname{HS}\{1,1\}\left(\beta=8 / t_{\mathrm{p}}\right)$ & 100 & 100 & 110 & 90 & 50 & 0.52 \\
\hline
\end{tabular}

the case for rectangular and Gaussian pulses, which always require a proper adjustment of the pulse length, we assume the findings here to be rather generalisable. In order to discuss this more quantitatively, we simulated inversion profiles of the best performing pulses from Table 3 for different $B_{1}$ field strengths.

We compare the pulse profiles with $v_{1}=30 \mathrm{MHz}$, which corresponds to our setup with the cases where lower $\left(v_{1}=\right.$ $20 \mathrm{MHz})$ or higher $\left(v_{1}=40 \mathrm{MHz}\right) B_{1}$ field strengths are reached. Figure 6 shows how the different pulses behave when different $B_{1}$ field strengths are used. The WURST pulse (Fig. 6b) shows the least variation for different $B_{1}$ field strengths. As expected, the inversion efficiency drops a little bit for $v_{1}=20 \mathrm{MHz}$. But this drop seems to be rather insignificant, and good modulation depths can still be expected. The decrease in inversion efficiency is a bit more significant for the HS $\{1,6\}$ pulse, so that a small reduction in the modulation depth is possible here. Both pulse profiles do not show significant changes when a higher $B_{1}$ field strength is used. The HS $\{1,1\}$ pulse has a massive drop in inversion efficiency when going to lower $B_{1}$ field strengths. This does not come as a surprise as the inversion efficiency is already incomplete at $v_{1}=30 \mathrm{MHz}$. Here, it might be advantageous to reduce the $\beta$ parameter of the $\operatorname{HS}\{1,1\}$ pulse. As has been stated earlier, this will increase the inversion efficiency. For a higher $B_{1}$ field strength of $v_{1}=40 \mathrm{MHz}$ the inversion efficiency of this $\operatorname{HS}\{1,1\}$ will increase. Therefore, a higher modulation depth comparable to the HS $\{1,1\}$ pulse is expected. As this will also increase the background decay, a higher MNR is not guaranteed. The chirp pulse also shows a rather strong decrease in the inversion efficiency for a $v_{1}=20 \mathrm{MHz}$. However, the inversion efficiency also decreased for a higher $B_{1}$ field strength of $v_{1}=40 \mathrm{MHz}$. This rather unexpected behaviour is probably caused by an insufficient smoothing of the edges of the chirp pulse. With higher $B_{1}$ field strength the initial effective magnetic field vector in the accelerated frame becomes less aligned with the $z$ axis. Therefore, smoothing becomes more important. In Fig. S10, we compared the inversion profiles of 36 and $100 \mathrm{~ns}$ chirp pulses with and without quarter sine smoothing. When quarter sine smoothing is applied, chirp pulses can with a length of $36 \mathrm{~ns}$ indeed reach a high inversion efficiency with $v_{1}=40 \mathrm{MHz}$. (Fig. S10b). As the width of the inversion profile of this chirp pulse drops significantly for smaller $B_{1}$ field strengths, it is only advisable to implement a quarter sine smoothing with chirp pulses of a length of $36 \mathrm{~ns}$ when enough microwave power is available. The situation looks different for chirp pulses with a pulse length of $100 \mathrm{~ns}$. Here, the inversion profile looks very similar for all tested $B_{1}$ field strengths. Particularly for smaller $B_{1}$ field strengths we expect $100 \mathrm{~ns}$ chirp pulses to outperform chirp pulses with a length of $36 \mathrm{~ns}$.

Another crucial parameter for DEER measurements that can vary from setup to setup is the width of the resonator profile. Here, we have a FWHM of approximately $200 \mathrm{MHz}$. Larger widths do not seem to be necessary because they would exceed the width of the spectrum of the nitroxide. If only a smaller width is available, the offset between pump and observer pulses might need to be reduced. This would increase the overlap between the observer and pump pulses. This problem could be overcome by either using longer pump pulses or reducing the frequency width of the broadbandshaped pulses. As a narrower resonator profile is also necessarily steeper, it might also be necessary to perform a resonator bandwidth compensation as suggested by Doll et al. (2013). Performing a resonator bandwidth compensation with our setup does not give a significant advantage in the $\eta_{2 P}$ value (see Sect. S15). This is probably due to the rather flat resonator profile in the region with maximum sensitivity where the pump pulse is applied.

\subsection{Background behaviour}

The broader excitation profile of broadband-shaped pulses will increase the background decay, which results in a higher noise level of broadband-shaped pump pulses compared to rectangular or Gaussian pump pulses. We find an approximately linear relation between the modulation depth and the background decay (see Sect. S19). To investigate this effect more deeply, we evaluated the MNR of the experimental DEER form factors, excluding the later part of the form factor and only taking into account the first part up to a truncation time $\tau_{\text {truncation. }}$. Truncation of the DEER trace will not change the modulation depth, but due to the background decay, the noise level will be different. Figure 7 shows the MNR of broadband pulses as a function of the truncation 

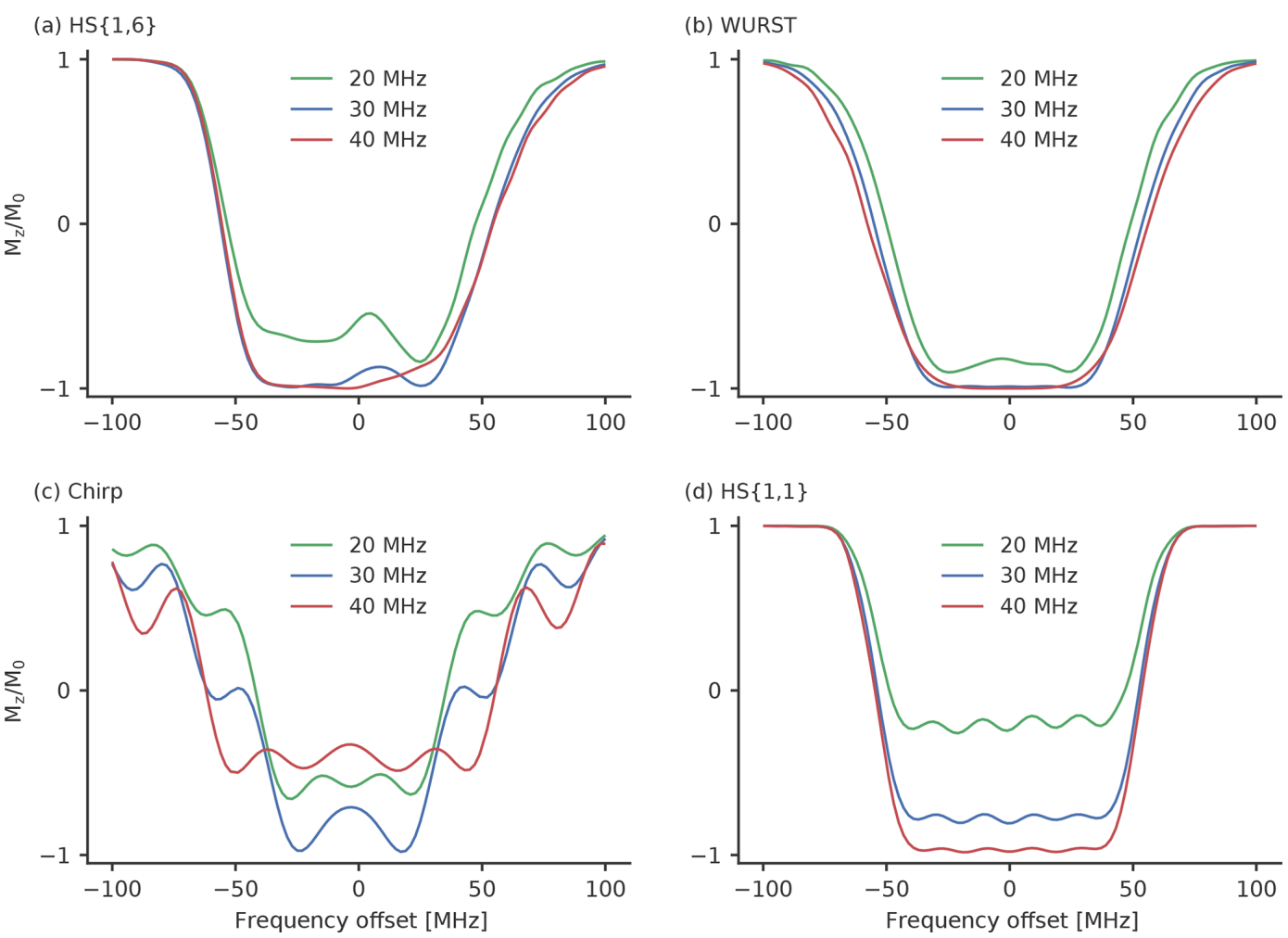

Figure 6. The inversion profiles of the best performing (a) $\operatorname{HS}\{1,6\}$, (b) WURST, (c) chirp and (d) HS $\{1,1\}$ pulses with the parameters from Table 3. They were simulated with a $B_{1}$ field strength of $v_{1}=20 \mathrm{MHz}$ (green), $30 \mathrm{MHz}$ (blue) and $40 \mathrm{MHz}$ (red). These field strengths correspond to $\pi$-pulse lengths of 25.0, 16.7 (which approximately corresponds to our setup) and $12.5 \mathrm{~ns}$. The $B_{1}$ field here is depicted as the Rabi frequency.

time $\tau_{\text {truncation. }}$ As expected, the MNR decreases with increasing $\tau_{\text {truncation }}$ for all pulses, because of the increase in the noise. However, the rate of the decrease in MNR is different for different pulse types, which means that the relative performance of the pulses also depends on the length of the DEER trace and therefore on the distance between the spin centres that is supposed to be measured.

It turns out that HS $\{1,6\}$ and WURST pulses with their higher modulation depths have the highest MNR for short DEER traces, whereas for longer traces HS $\{1,1\}$ and chirp pulses are better. The background decay seems to play a decisive role for the MNR and the pulses resulting in a high modulation depth also have a larger background decay. As the background decay causes the noise level to increase with increasing dipolar evolution time, its influence is less pronounced for short DEER traces, where the high modulation depth seems to be leading to a high MNR. For longer traces, a high modulation depth is linked to a strong background decay and a high noise level towards the end of the trace. Therefore, the MNR of pump pulses generating a high modulation depth decreases more strongly than for pulses effecting a smaller modulation depth. This means that HS $\{1,1\}$ and chirp pulses perform better for longer traces.

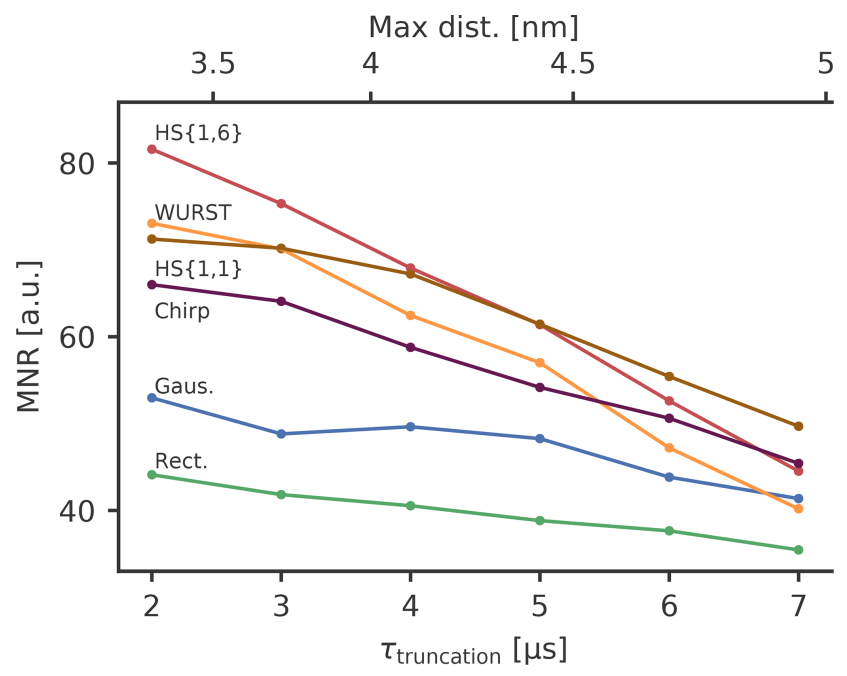

Figure 7. The MNR value as a function of the dipolar evolution time up to which the noise has been evaluated, i.e. the truncation time $\tau_{\text {truncation. }}$ The sample has a concentration of $80 \mu \mathrm{M}$ of the spin-labelled ligand. The maximum distance according to Eq. (13) is depicted in the upper $x$ axis. The line between the points is only a guide for the eyes. 
As rectangular and Gaussian pump pulses have rather small modulation depths, the corresponding decrease in the MNR due to the background decay is also rather small, which means that the improvement achievable with broadbandshaped pulses is greater for shorter DEER traces. For short truncation times $\tau_{\text {truncation }}$ of $2 \mu \mathrm{s}$ we observe an increase in MNR from 44 for rectangular pulses (RR) to 82 for the best broadband-shaped pulse (RS), which was an $\operatorname{HS}\{1,6\}$ pulse in this case. This corresponds to an increase of $86 \%$. For long truncation times $\tau_{\text {truncation }}$ of $7 \mu \mathrm{s}$, this increase goes down to $43 \%$. This means that the MNR improvement that can be achieved by broadband-shaped pulses can be drastically dependent on the length of the measured DEER trace and therefore on the distance range to be covered by the measurement. For a concentration of $80 \mu \mathrm{M}$, a high MNR improvement can be achieved if the maximum distance of interest is below $4 \mathrm{~nm}$ with a pulse that achieves a high modulation depth. This would correspond to the HS $\{1,6\}$ and WURST pulse in this case. If longer distances up to $5 \mathrm{~nm}$ are to be detected, it seems to be advantageous to use pulses that might not give the highest modulation depth in order to reduce the background decay. An extrapolation for higher truncation times shows that if even longer distances are of interest, broadbandshaped pulses will not give a better MNR compared to rectangular pulses. Here, it is necessary to reduce the background decay by using lower concentrations.

The performance of all the pulses at $\tau_{\text {truncation }}=2 \mu$ s can be found in Tables S7-S10. The chirp, WURST, HS $\{1,1\}$ and HS $\{1,6\}$ pulse resulting in the best MNR are summarised in Table 4. For the broadband-shaped pulses there were some minor changes in the parameters that gave the MNR when the truncation time was set to a shorter value of $t_{\text {truncation }}=2 \mu \mathrm{s}$. For RR and GG there were changes in the best parameter settings.

\subsection{Concentration dependence}

To check for a concentration-dependent performance of broadband-shaped pulses, we also prepared a sample with a lower concentration of $30 \mu \mathrm{M}$ ligand and $60 \mu \mathrm{M}$ WGA and performed DEER measurements with the optimised parameter settings for the short chirp, WURST, $\operatorname{HS}\{1,1\}$ and HS $\{1,6\}$ pulses. We did, however, not check observer frequencies of $33.91 \mathrm{GHz}$, since they always performed worse than an observer position of $33.93 \mathrm{GHz}$. For RR we tested an offset of $70 \mathrm{MHz}$ and $60 \%$ intensity; for GG we tested an offset of $70 \mathrm{MHz}$ as well, but an intensity of $100 \%$, as these settings performed best before.

This sample showed almost no background for all used pump pulses (see Fig. S17b). As the influence of the background is minimised due to the low concentration, we expected to find the trends in the MNR as for the case of the high concentrated samples and short truncation times. Figure 8 shows the MNR as a function of the truncation time point $\tau_{\text {truncation }}$ up to which the noise has been evaluated. As

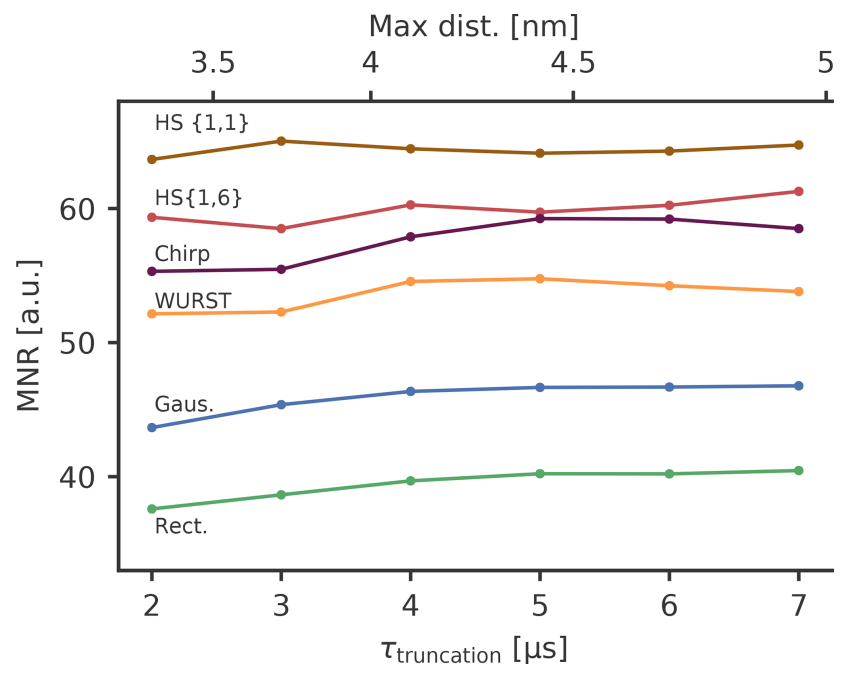

Figure 8. The MNR as a function of the dipolar evolution time up to which the noise has been evaluated. The sample has a concentration of $30 \mu \mathrm{M}$ of the spin-labelled ligand. The maximum distance according to Eq. (13) is depicted in the upper $x$ axis. The line between the points is only a guide for the eyes.

expected, no significant decrease in the MNR with higher truncation times $\tau_{\text {truncation }}$ was found. Without a significant background the noise towards the end of the backgroundcorrected form factor does not increase significantly. The decrease in the MNR found for the high concentration sample was therefore not observed here. For some pulses there is a slight increase in the MNR with the truncation time; however, we attributed this behaviour to a numerical uncertainty in the analysis.

With the low concentration sample, we found an MNR of 40 and a modulation depth of $31 \%$ for rectangular pulses; for Gaussian pulses we found an MNR of 47 and a modulation depth of $30 \%$. Thus, also at lower spin concentrations the Gaussian pulses lead to a similar modulation depth to rectangular pulses, but again to an overall higher MNR. Table 5 shows the results for the different broadband-shaped pump pulses in combination with the observer pulse with which they performed best.

Table 5 shows the optimised parameters for the different pump pulses. All results can be found in Table S11. The parameters found for the observer and pump pulses differ slightly from the parameters identified for the high concentration sample, but lie in a similar range.

The broadband-shaped pump pulses resulted in a modulation depth that is a bit lower than for the sample with the high concentration. The MNR was lower as well. Furthermore, the order of performance of the different pulse types changed. While we expected $\operatorname{HS}\{1,6\}$ and WURST pulses with their high modulation depths to perform better than $\operatorname{HS}\{1,1\}$ and chirp pulses for a sample less susceptible to background influence, $\operatorname{HS}\{1,1\}$ pulses actually performed 
Table 4. The parameters of the observer and pump pulses resulting in the best MNR for each pulse type when the MNR was evaluated up to $\tau_{\text {truncation }}=2 \mu \mathrm{s}$. All observer pulses are Gaussian pulses with a pulse length of $74 \mathrm{~ns}$ for $60 \%$ intensity and $56 \mathrm{~ns}$ for $100 \%$ intensity. The observer frequency was $33.93 \mathrm{GHz}$ for all pulses.

\begin{tabular}{lrrrrrr}
\hline Pump pulse & $\begin{array}{r}\text { Obs. } \\
\text { Amp. }(\%)\end{array}$ & $\begin{array}{r}t_{\pi} \\
(\mathrm{ns})\end{array}$ & $\begin{array}{r}\Delta f \\
(\mathrm{MHz})\end{array}$ & $\begin{array}{r}\text { Offset } \\
(\mathrm{MHz})\end{array}$ & MNR & Mod depth $\lambda$ \\
\hline HS $\{1,6\}\left(\beta=10 / t_{\mathrm{p}}\right)$ & 60 & 100 & 110 & 90 & 82 & 0.61 \\
WURST $(n=6)$ & 100 & 100 & 160 & 90 & 73 & 0.63 \\
Chirp (no smoothing) & 100 & 36 & 120 & 80 & 65 & 0.49 \\
HS $\{1,1\}\left(\beta=8 / t_{\mathrm{p}}\right)$ & 60 & 100 & 110 & 80 & 74 & 0.52 \\
\hline
\end{tabular}

Table 5. The parameters of the observer and pump pulses resulting in the best MNR for each pulse type. All observer pulses are Gaussian pulses with pulse lengths of $74 \mathrm{~ns}$ for $60 \%$ intensity and $56 \mathrm{~ns}$ for $100 \%$ intensity. The observer frequency was $33.93 \mathrm{GHz}$ for all pulses. The MNR was evaluated up to $7 \mu \mathrm{s}$.

\begin{tabular}{lrrrrrr}
\hline Pump pulse & $\begin{array}{r}\text { Obs. } \\
\text { Amp. }(\%)\end{array}$ & $\begin{array}{r}t_{\pi} \\
(\mathrm{ns})\end{array}$ & $\begin{array}{r}\Delta f \\
(\mathrm{MHz})\end{array}$ & $\begin{array}{r}\text { Offset } \\
(\mathrm{MHz})\end{array}$ & MNR & Mod depth $\lambda$ \\
\hline HS $\{1,6\}\left(\beta=10 / t_{\mathrm{p}}\right)$ & 100 & 100 & 110 & 90 & 61 & 0.55 \\
WURST $(n=6)$ & 60 & 100 & 160 & 100 & 54 & 0.59 \\
Chirp (no smoothing) & 100 & 36 & 120 & 80 & 58 & 0.46 \\
HS $\{1,1\}\left(\beta=8 / t_{\mathrm{p}}\right)$ & 100 & 100 & 110 & 90 & 65 & 0.47 \\
\hline
\end{tabular}

best and WURST pulses were the worst broadband-shaped pulses. HS $\{1,1\}$ pulses lead to an increase in the MNR of $60 \%$ compared to rectangular pulses. This is also lower than the $86 \%$ increase that was obtained for the $80 \mu \mathrm{M}$ ligand concentration. The reason for the change in this behaviour is probably a difference in the resonator profile that we noticed compared to the other sample with the higher concentration (see Sect. S22). The achieved $B_{1}$ field was a bit lower for this sample, which changes the performance of the pulses. However, $\operatorname{HS}\{1,1\}$ and $\operatorname{HS}\{1,6\}$ pulses both give a good MNR with a high concentration as well as with a low concentration.

When the MNR is to be increased by using broadbandshaped pulses to detect long distances $>5 \mathrm{~nm}$, lower concentrations are preferable as they reduce the enhancement of the background decay. Here, switching to a concentration of $30 \mu \mathrm{M}$ of the doubly labelled ligand was enough to significantly reduce the influence of the background. In Sect. S19 we performed analytical calculations to estimate the potential MNR increase that can be achieved by switching to broadband-shaped pulses for different concentrations and distance ranges. For maximum distances below $4 \mathrm{~nm}$ an increase in the MNR can be expected for all concentrations up to approximately $100 \mu \mathrm{M}$. The situation is different if distances over $6 \mathrm{~nm}$ are to be detected. A significant gain can only be expected for smaller concentrations in the range between 10 and $30 \mu \mathrm{M}$. For higher concentrations the MNR gain drops quickly. For higher concentrations in the range of $80 \mu \mathrm{M}$ a MNR decrease has to be expected in this distance regime. This is discussed in more detail in Sect. S21.

\section{Conclusion and outlook}

We have compared various broadband-shaped pulses as pump pulses for DEER spectroscopy in $Q$ band performed on samples with nitroxide spin labels and investigated under which circumstances they perform best. By increasing the inversion profile, broadband-shaped pulses can increase the modulation depth from $30 \%$ with rectangular pulses up to $60 \%$. However, with a larger inversion profile of broadbandshaped pulses the overlap with the observer pulse and the background decay will also increase. Both of those effects will tend to reduce the MNR. The overall MNR increase will therefore be a compromise between the increase in the modulation depth and the smaller echo and larger background contribution.

Systematic analysis of a trial-and-error optimisation has shown that the performance of broadband-shaped pulses depends on the dipolar evolution time and the concentration of spin centres. Larger dipolar evolution times mean that the background has decayed more strongly by the end of the form factor. Pulses with a higher inversion efficiency will produce a larger background decay and their performance decreases more strongly for longer traces than for pulses with a smaller inversion efficiency. We found $\operatorname{HS}\{1,1\}$ and $\mathrm{HS}\{1,6\}$ in combination with Gaussian observer pulses to give a good MNR for high as well as low spin concentrations. HS $\{1,1\}$ have a lower inversion efficiency and therefore a lower modulation depth, but they perform better with longer traces needed for longer distances. The exact parameters depend on the setup, but with values of $\beta=8 / t_{\mathrm{p}}$ or $\beta=10 / t_{\mathrm{p}}, t_{\mathrm{p}}=100 \mathrm{~ns}$, and $\Delta f=110 \mathrm{MHz}$ and an offset of 
80 or $90 \mathrm{MHz}$ we typically achieved good results. If a high modulation depth which is particularly suitable for short distances should be achieved, $\operatorname{HS}\{1,6\}$ and WURST pulses are the best pulses. Good parameters are $\beta=10 / t_{\mathrm{p}}, t_{\mathrm{p}}=100 \mathrm{~ns}$, $\Delta f=110 \mathrm{MHz}$ and an offset of $90 \mathrm{MHz}$ for $\operatorname{HS}\{1,6\}$ and $n=6, t_{\mathrm{p}}=100 \mathrm{~ns}, \Delta f=160 \mathrm{MHz}$ and an offset of 90 or $100 \mathrm{MHz}$ for WURST pulses.

Data availability. The raw data can be downloaded at https://doi.org/10.5281/zenodo.3726735 (Scherer et al., 2020).

Supplement. The supplement related to this article is available online at: https://doi.org/10.5194/mr-1-59-2020-supplement.

Author contributions. AS, ST, SW and MD conceived the research idea and designed the conducted experiments. AS conducted the EPR experiments and analysed the results with ST. The spinlabelled ligand was synthesised in the lab of VW. AS prepared all the figures and wrote the draft manuscript. All the authors discussed the results and revised the manuscript.

Competing interests. The authors declare that they have no conflict of interest.

Acknowledgements. We thank Philipp Rohse for the synthesis of the spin-labelled ligand. Jörg Fischer is thanked for sample preparation. This project received funding from the European Research Council (ERC) under the European Union's Horizon 2020 research and innovation programme (grant agreement number: 772027 SPICE - ERC-2017-COG). Andreas Scherer is grateful for financial support from the Zukunftskolleg of the University of Konstanz. Andreas Scherer, Sonja Tischlik and Sabrina Weickert are grateful for financial support from the Konstanz Research School Chemical Biology (KoRS-CB).

Financial support. This work was supported financially by the European Research Council (ERC) under the European Union's Horizon 2020 research and innovation programme (grant no. 772027-SPICE-ERC-2017-COG).

Review statement. This paper was edited by Daniella Goldfarb and reviewed by two anonymous referees.

\section{References}

Abdullin, D., Duthie, F., Meyer, A., Müller, E. S., Hagelueken, G., and Schiemann, O.: Comparison of PELDOR and RIDME for Distance Measurements between Nitroxides and LowSpin Fe(III) Ions, J. Phys. Chem. B, 119, 13534-13542, https://doi.org/10.1021/acs.jpcb.5b02118, 2015.
Bahrenberg, T., Rosenski, Y., Carmieli, R., Zibzener, K., Qi, M., Frydman, V., Godt, A., Goldfarb, D., and Feintuch, A.: Improved sensitivity for W-band $\mathrm{Gd}(\mathrm{III})-\mathrm{Gd}(\mathrm{III})$ and nitroxide-nitroxide DEER measurements with shaped pulses, J. Magn. Reson., 283, 1-13, https://doi.org/10.1016/j.jmr.2017.08.003, 2017.

Baum, J., Tycko, R., and Pines, A.: Broadband and adiabatic inversion of a two-level system by phase-modulated pulses, Phys. Rev. A, 32, 3435-3447, 1985.

Böhlen, J.-M. and Bodenhausen, G.: Experimental Aspects of Chirp NMR Spectroscopy, J. Magn. Reson. A, 102, 293-301, https://doi.org/10.1006/jmra.1993.1107, 1993.

Borbat, P. P., Georgieva, E. R., and Freed, J. H.: Improved Sensitivity for Long-Distance Measurements in Biomolecules: FivePulse Double Electron-Electron Resonance, J. Phys. Chem. Lett., 4, 170-175, https://doi.org/10.1021/jz301788n, 2013.

Breitgoff, F. D., Soetbeer, J., Doll, A., Jeschke, G., and Polyhach, Y. O.: Artefact suppression in 5-pulse double electron electron resonance for distance distribution measurements, Phys. Chem. Chem. Phys., 19, 15766-15779, 2017.

Breitgoff, F. D., Keller, K., Qi, M., Klose, D., Yulikov, M., Godt, A., and Jeschke, G.: UWB DEER and RIDME distance measurements in $\mathrm{Cu}(\mathrm{II})-\mathrm{Cu}(\mathrm{II})$ spin pairs, J. Magn. Reson., 308, 106560106578, https://doi.org/10.1016/j.jmr.2019.07.047, 2019.

Collauto, A., Feintuch, A., Qi, M., Godt, A., Meade, T., and Goldfarb, D.: Gd(III) complexes as paramagnetic tags: Evaluation of the spin delocalization over the nuclei of the ligand, J. Magn. Reson., 263, 156-163, https://doi.org/10.1016/j.jmr.2015.12.025, 2016.

Dalaloyan, A., Qi, M., Ruthstein, S., Vega, S., Godt, A., Feintuch, A., and Goldfarb, D.: Gd(iii)-Gd(iii) EPR distance measurements - the range of accessible distances and the impact of zero field splitting, Phys. Chem. Chem. Phys., 17, 18464-18476, https://doi.org/10.1039/C5CP02602D, 2015.

Deschamps, M., Kervern, G., Massiot, D., Pintacuda, G., Emsley, L., and Grandinetti, P. J.: Superadiabaticity in magnetic resonance, J. Chem. Phys., 129, 204110, https://doi.org/10.1063/1.3012356, 2008.

Di Valentin, M., Albertini, M., Zurlo, E., Gobbo, M., and Carbonera, D.: Porphyrin Triplet State as a Potential Spin Label for Nanometer Distance Measurements by PELDOR Spectroscopy, J. Am. Chem. Soc., 136, 6582-6585, https://doi.org/10.1021/ja502615n, 2014.

Doll, A. and Jeschke, G.: Wideband frequency-swept excitation in pulsed EPR spectroscopy, Spec. Issue Methodol. Adv. EPR Spectrosc. Imaging, 280, 46-62, https://doi.org/10.1016/j.jmr.2017.01.004, 2017.

Doll, A., Pribitzer, S., Tschaggelar, R., and Jeschke, G.: Adiabatic and fast passage ultra-wideband inversion in pulsed EPR, J. Magn. Reson., 230, 27-39, https://doi.org/10.1016/j.jmr.2013.01.002, 2013.

Doll, A., Qi, M., Wili, N., Pribitzer, S., Godt, A., and Jeschke, G.: Gd(III)-Gd(III) distance measurements with chirp pump pulses, J. Magn. Reson., 259, 153-162, https://doi.org/10.1016/j.jmr.2015.08.010, 2015.

Doll, A., Qi, M., Godt, A., and Jeschke, G.: CIDME: Short distances measured with long chirp pulses, J. Magn. Reson., 273, 73-82, https://doi.org/10.1016/j.jmr.2016.10.011, 2016. 
Edwards, T. H. and Stoll, S.: Optimal Tikhonov regularization for DEER spectroscopy, J. Magn. Reson., 288, 58-68, https://doi.org/10.1016/j.jmr.2018.01.021, 2018.

Fábregas Ibáñez, L., and Jeschke, G.: Optimal background treatment in dipolar spectroscopy, Phys. Chem. Chem. Phys., 22, 1855-1868, https://doi.org/10.1039/C9CP06111H, 2020.

Garwood, M. and DelaBarre, L.: The Return of the Frequency Sweep: Designing Adiabatic Pulses for Contemporary NMR, J. Magn. Reson., 153, 155-177, https://doi.org/10.1006/jmre.2001.2340, 2001.

Grytz, C. M., Kazemi, S., Marko, A., Cekan, P., Guntert, P., Sigurdsson, S. T., and Prisner, T. F.: Determination of helix orientations in a flexible DNA by multi-frequency EPR spectroscopy, Phys. Chem. Chem. Phys., 19, 29801-29811, https://doi.org/10.1039/C7CP04997H, 2017.

Hintze, C., Bücker, D., Domingo Köhler, S., Jeschke, G., and Drescher, M.: Laser-Induced Magnetic Dipole Spectroscopy, J. Phys. Chem. Lett., 7, 2204-2209, https://doi.org/10.1021/acs.jpclett.6b00765, 2016.

$\mathrm{Hu}, \mathrm{P}$. and Hartmann, S. R.: Theory of spectral diffusion decay using an uncorrelated-sudden-jump model, Phys. Rev. B, 9, 1-13, https://doi.org/10.1103/PhysRevB.9.1, 1974.

Hubbell, W. L., Gross, A., Langen, R., and Lietzow, M. A.: Recent advances in site-directed spin labeling of proteins, Curr. Opin. Struct. Biol., 8, 649-656, https://doi.org/10.1016/S0959440X(98)80158-9, 1998.

Jassoy, J. J., Meyer, A., Spicher, S., Wuebben, C., and Schiemann, O.: Synthesis of Nanometer Sized Bis-and Tris-trityl Model Compounds with Different Extent of Spin-Spin Coupling, Molecules, 23, 682-699, 2018.

Jeschke, G.: Instrumentation and experimental setup, in: ESR spectroscopy in membrane biophysics, vol. 27, edited by: Hemminga, M. A. and Berliner, L., pp. 17-47, Springer Science \& Business Media, New York, USA, 2007.

Jeschke, G.: Dipolar Spectroscopy-Double-Resonance Methods, eMagRes, 5, 1459-1476, 2016.

Jeschke, G.: DEER Distance Measurements on Proteins, Annu. Rev. Phys. Chem., 63, 419-446, https://doi.org/10.1146/annurevphyschem-032511-143716, 2012.

Jeschke, G., Bender, A., Paulsen, H., Zimmermann, H., and Godt, A.: Sensitivity enhancement in pulse EPR distance measurements, J. Magn. Reson., 169, 1-12, https://doi.org/10.1016/j.jmr.2004.03.024, 2004.

Jeschke, G., Chechik, V., Ionita, P., Godt, A., Zimmermann, H., Banham, J., Timmel, C. R., Hilger, D., and Jung, H.: DeerAnalysis2006 - a comprehensive software package for analyzing pulsed ELDOR data, Appl. Magn. Reson., 30, 473-498, https://doi.org/10.1007/BF03166213, 2006.

Kobzar, K., Ehni, S., Skinner, T. E., Glaser, S. J., and Luy, B.: Exploring the limits of broadband $90^{\circ}$ and $180^{\circ}$ universal rotation pulses, J. Magn. Reson., 225, 142-160, https://doi.org/10.1016/j.jmr.2012.09.013, 2012.

Kupče, E. and Freeman, R.: Adiabatic Pulses for Wideband Inversion and Broadband Decoupling, J. Magn. Reson. A, 115, 273276, https://doi.org/10.1006/jmra.1995.1179, 1995a.

Kupče, E. and Freeman, R.: Stretched Adiabatic Pulses for Broadband Spin Inversion, J. Magn. Reson. A, 117, 246-256, https://doi.org/10.1006/jmra.1995.0750, 1995b.
Kupče, E. and Freeman, R.: Optimized Adiabatic Pulses for Wideband Spin Inversion, J. Magn. Reson. A, 118, 299-303, https://doi.org/10.1006/jmra.1996.0042, 1996.

Kuzhelev, A. A., Akhmetzyanov, D., Denysenkov, V. P., Krumkacheva, O., Shevelev, G., Bagryanskaya, E., and Prisner, T. F. F.: High-frequency pulsed electron-electron double resonance spectroscopy on DNA duplexes using trityl tags and shaped microwave pulses, Phys. Chem. Chem. Phys., 20, 26140 26144, https://doi.org/10.1039/C8CP03951H, 2018.

Lovett, J. E., Lovett, B. W., and Harmer, J.: DEER-Stitch: Combining three- and four-pulse DEER measurements for high sensitivity, deadtime free data, J. Magn. Reson., 223, 98-106, https://doi.org/10.1016/j.jmr.2012.08.011, 2012.

Mahawaththa, M., Lee, M., Giannoulis, A., Adams, L. A., Feintuch, A., Swarbrick, J., Graham, B., Nitsche, C., Goldfarb, D., and Otting, G.: Small Neutral Gd(III) Tags for Distance Measurements in Proteins by Double Electron-Electron Resonance Experiments, Phys. Chem. Chem. Phys., 20, 23535 23545, https://doi.org/10.1039/C8CP03532F, 2018.

Mentink-Vigier, F., Collauto, A., Feintuch, A., Kaminker, I., Tarle, V., and Goldfarb, D.: Increasing sensitivity of pulse EPR experiments using echo train detection schemes, J. Magn. Reson., 236, 117-125, https://doi.org/10.1016/j.jmr.2013.08.012, 2013.

Milikisiyants, S., Voinov, M. A., Marek, A., Jafarabadi, M., Liu, J., Han, R., Wang, S., and Smirnov, A. I.: Enhancing sensitivity of Double Electron-Electron Resonance (DEER) by using Relaxation-Optimized Acquisition Length Distribution (RELOAD) scheme, J. Magn. Reson., 298, 115-126, https://doi.org/10.1016/j.jmr.2018.12.004, 2019.

Milov, A. D., Salikhov, K. M., and Shirov, M. D.: Use of the double resonance in electron spin echo method for the study of paramagnetic center spatial distribution in solids., Fiz Tverd Tela, 23, 975-982, 1981.

Milov, A. D., Ponomarev, A. B., and Tsvetkov, Y. D.: Electronelectron double resonance in electron spin echo: Model biradical systems and the sensitized photolysis of decalin, Chem. Phys. Lett., 110, 67-72, https://doi.org/10.1016/0009-2614(84)801487, 1984

O'Dell, L. A.: The WURST kind of pulses in solid-state NMR, Solid State Nucl. Magn. Reson., 55-56, 28-41, https://doi.org/10.1016/j.ssnmr.2013.10.003, 2013.

Pannier, M., Veit, S., Godt, A., Jeschke, G., and Spiess, H.: Dead-Time Free Measurement of Dipole-Dipole Interactions between Electron Spins, J. Magn. Reson., 142, 331-340, https://doi.org/10.1006/jmre.1999.1944, 2000.

Polyhach, Y., Bordignon, E., Tschaggelar, R., Gandra, S., Godt, A., and Jeschke, G.: High sensitivity and versatility of the DEER experiment on nitroxide radical pairs at Q-band frequencies, Phys. Chem. Chem. Phys., 14, 10762-10773, https://doi.org/10.1039/C2CP41520H, 2012.

Pribitzer, S., Sajid, M., Hülsmann, M., Godt, A., and Jeschke, G.: Pulsed triple electron resonance (TRIER) for dipolar correlation spectroscopy, J. Magn. Reson., 282, 119-128, https://doi.org/10.1016/j.jmr.2017.07.012, 2017.

Robotta, M., Gerding, H. R., Vogel, A., Hauser, K., Schildknecht, S., Karreman, C., Leist, M., Subramaniam, V., and Drescher, M.: Alpha-Synuclein Binds to the Inner Membrane of Mitochondria in an $\alpha$-Helical Conformation, ChemBioChem, 15, 2499-2502, https://doi.org/10.1002/cbic.201402281, 2014. 
Rohse, P., Weickert, S., Drescher, M., and Wittmann, V.: Precipitation-Free High-Affinity Multivalent Binding by Inline Lectin Ligands, Chem. Sci., accepted, https://doi.org/10.1039/D0SC01744B, 2020.

Scherer, A., Tischlik, S., Weickert, S., Wittmann, V., and Drescher, M.: Raw data for "Optimising broadband pulses for DEER depends on concentration and distance range of interest, Zenodo, https://doi.org/10.5281/zenodo.3726735, 2020.

Schwefel, D., Maierhofer, C., Beck, J. G., Seeberger, S., Diederichs, K., Möller, H. M., Welte, W., and Wittmann, V.: Structural Basis of Multivalent Binding to Wheat Germ Agglutinin, J. Am. Chem. Soc., 132, 8704-8719, https://doi.org/10.1021/ja101646k, 2010.

Spindler, P. E., Glaser, S. J., Skinner, T. E., and Prisner, T. F.: Broadband Inversion PELDOR Spectroscopy with Partially Adiabatic Shaped Pulses, Angew. Chem. Int. Ed., 52, 3425-3429, https://doi.org/10.1002/anie.201207777, 2013.

Spindler, P. E., Schöps, P., Kallies, W., Glaser, S. J., and Prisner, T. F.: Perspectives of shaped pulses for EPR spectroscopy, J. Magn. Reson., 280, 30-45, https://doi.org/10.1016/j.jmr.2017.02.023, 2017.
Tait, C. E. and Stoll, S.: Coherent pump pulses in Double Electron Electron Resonance spectroscopy, Phys. Chem. Chem. Phys., 18, 18470-18485, https://doi.org/10.1039/C6CP03555H, 2016.

Tannús, A. and Garwood, M.: Improved Performance of Frequency-Swept Pulses Using Offset-Independent Adiabaticity, J. Magn. Reson. A, 120, 133-137, https://doi.org/10.1006/jmra.1996.0110, 1996.

Teucher, M. and Bordignon, E.: Improved signal fidelity in 4-pulse DEER with Gaussian pulses, J. Magn. Reson., 296, 103-111, https://doi.org/10.1016/j.jmr.2018.09.003, 2018.

Wort, J., Ackermann, K., Giannoulis, A., Stewart, A., Norman, D., and Bode, B. E.: Sub-micromolar pulse dipolar EPR spectroscopy reveals increasing CuII-labelling of double-histidine motifs with lower temperature, Angew. Chem., 131, 1180711811, https://doi.org/10.1002/ange.201904848, 2019. 